\title{
Implementation of Biorthogonal Wavelet Transform Using Windowed APDF Based on DCT
}

\author{
Xiao Zhou, Qiming Fu, Fanfan Yang and Chengyou Wang* \\ School of Mechanical, Electrical and Information Engineering, Shandong University, \\ Weihai 264209, China \\ zhouxiao@sdu.edu.cn,fqmsdu@163.com,yangfanfanwh@163.com, \\ wangchengyou@sdu.edu.cn
}

\begin{abstract}
All phase digital filter (APDF) is a new type of linear phase FIR digital filter that was proposed in recent years. Firstly, the theory of biorthogonal wavelet transform and windowed APDF based on DCT is expounded and the relationship between them is discussed. Secondly, a novel algorithm is proposed to implement biorthogonal wavelet transform by using windowed APDF based on DCT. As an important application of biorthogonal wavelet transform, multi-resolution analysis of 2-D image signal could be used to test the feasibility and applicability of the proposed algorithm. Finally, the simulation results using MATLAB tool are shown in this paper and the analysis indicates that the proposed method performs well.
\end{abstract}

Keywords: DCT Filtering; Windowed All Phase Digital Filter; Biorthogonal Wavelet Transform; Mallat Algorithm

\section{Introduction}

The design of linear phase FIR digital filter was proposed in the last century. Due to its well-developed theories and easy implementation by fast Fourier transform (FFT), it has been playing an important role in image filtering. There are several methods for designing FIR digital filters, they are the windowing, the frequency sampling and the equal ripple approximation [1]. The equal ripple approximation can not precisely control the responses at specified frequencies, and the designing process is complex to some extent. The frequency sampling usually requires inserting some transition points to improve the ripple; consequently the cut-off frequency is controlled not easily. The windowing requires much more frequency samples in order to give a desired impulse response truncated by a window. While the windowing method improving ripples, it induces a wider transition band and loses control of the frequency response at the sampled points as well. Because the conventional filter designing methods have many disadvantages, some scholars try to improve it. Combining with overlapped digital filtering, several kinds of all phase digital filters based on the discrete Fourier transform (DFT), discrete cosine transform (DCT) and inverse DCT (IDCT) were proposed [2-5]. The all phase digital filter method is a new scheme for FIR filter design, and it is superior to the conventional methods in overall filter performance. Also, we can further improve the performance of the filter by adding a front window and a rear window on the input signal and output signal respectively. In the following sections of this paper, the filter designed by the method of all phase is called all phase digital filter (APDF) in a precise manner. 
Compared with FFT, DCT has many advantages. Because the DCT transform matrix easily reflects the characteristics of image signals and human speech signals, DCT is considered as the quasi-perfect transform [6]. DCT is the most widely used orthogonal transform, which is still a hot research topic. However, DCT also has many disadvantages such as blocking artifacts and nonlinear phase characteristics. Therefore, the DCT filter is also in the process of development. The all phase digital filters based on DCT have many advantages [7], for example, since we can avoid complex operation in the DCT domain, the sequency response vector is not required to be real symmetric. Therefore, more APDFs can be designed based on DCT than that based on DFT.

As an important form of wavelet analysis, the biorthogonal wavelet transform has a lot of advantages. For example, the wavelet filter banks are symmetrical with compact support [8]. Now, biorthogonal wavelet transform has been used widely in the fields of image denoising [9] and image coding [10]. In 1989, pyramid algorithm was proposed by Mallat to implement fast wavelet transform (FWT) [11], and it is equivalent to the importance of FFT in Fourier transform. In 2013, Jiang et al. [12] proposed an algorithm to realize biorthogonal wavelet transform using discrete cosine sequency filter (DCSF). With analyzing the biorthogonal wavelet transform implemented by Mallat algorithm and windowed APDF based on DCT, we propose a new algorithm to implement biorthogonal wavelet transform using windowed APDF based on DCT.

The rest of this paper is organized as follows. Section 2 starts with a brief review of biorthogonal wavelet transform, and then the bior4.4 biorthogonal wavelet is taken as an example to illustrate the transform. Windowed APDF based on DCT is introduced in Section 3. Section 4 presents the algorithm designed to implement biorthogonal wavelet transform using the windowed APDF based on DCT. The proposed method is tested by typical image, and the experimental results are given in Section 5. Finally, conclusion and future work are given finally in Section 6.

\section{Biorthogonal Wavelet Transform}

The biorthogonal wavelet transform is composed of the decomposition process and the reconstruction process with two different wavelets $\psi(t)$ and $\tilde{\psi}(t) . \psi(t)$ is used in the decomposition process, and $\tilde{\psi}(t)$ is used in the reconstruction process. $\psi(t)$ and $\tilde{\psi}(t)$ are dual and orthogonal to each other, and this relationship is called biorthogonal.

Meanwhile, there are two scale functions $\phi(t)$ and $\tilde{\phi}(t)$ in the above processes, these two scale functions are also dual and orthogonal. Each of them can be used in the decomposition process, and the other one can be used in the reconstruction process.

The biorthogonal transform can be easily implemented using Mallat algorithm, which is shown in Figure 1. There are two pairs of sequence, $\left(\left\{\tilde{p}_{k}\right\},\left\{\tilde{q}_{k}\right\}\right)$ and $\left(\left\{p_{k}\right\},\left\{q_{k}\right\}\right)$. The $\left\{\tilde{p}_{k}\right\}$ and $\left\{\tilde{q}_{k}\right\}$ are called decomposition sequences, the $\left\{p_{k}\right\}$ and $\left\{q_{k}\right\}$ are called reconstruction sequences. Sliding inner product is introduced in [13].

What we can conclude from Figure 1 is that the essence of Mallat algorithm is filtering the signal $\left\{c_{N, k}\right\}$ by decomposition filters $\left\{\tilde{p}_{k}\right\}$ and $\left\{\tilde{q}_{k}\right\}$. Then the results are down-sampled by factor 2 . After the decomposition, the original signal is divided into two parts with the length which is equal to half of the original length. One part is the signal $\left\{c_{N-1, k}\right\}$ generated by low-pass filter $\left\{\tilde{p}_{k}\right\}$, which can be seen as the approximation of the original signal. And the other part is the signal $\left\{d_{N-1, k}\right\}$ generated by high-pass filter $\left\{\tilde{q}_{k}\right\}$, which can be seen as the detail of the original signal. On the 
contrary, the reconstruction process takes the reverse process to reconstruct the original signal by reconstruction filters $\left\{p_{k}\right\}$ and $\left\{q_{k}\right\}$.

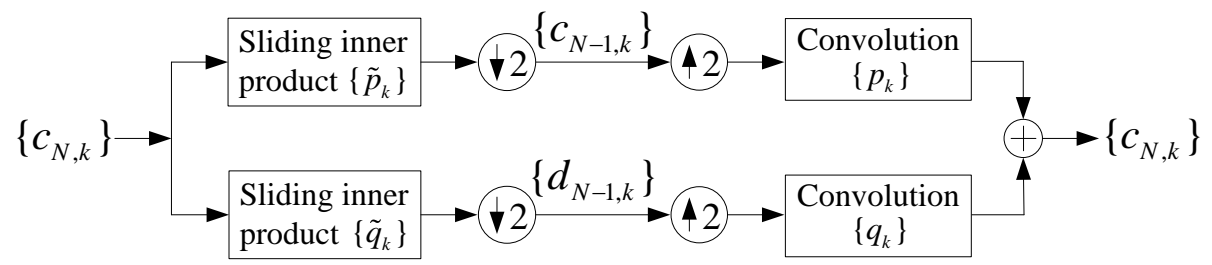

\section{Figure 1. Decomposition and Reconstruction Processes of 1-D Mallat Algorithm}

Reference [13] presented that realizing the wavelet multi-resolution analysis for twodimensional signal is similar to that of one-dimensional signal. We denote $\left\{c_{N-1, k}\right\}$ and $\left\{d_{N-1, k}^{i}(i=1,2,3)\right\}$ as the approximation and detail of 2-D signal at scale $N-1$ respectively. After the horizontal and vertical filtering processing to the twodimensional signal $\left\{c_{N, k}\right\}$, four different frequency bands $\left\{c_{N-1, k}\right\},\left\{d_{N-1, k}^{1}\right\},\left\{d_{N-1, k}^{2}\right\}$, and $\left\{d_{N-1, k}^{3}\right\}$ could be obtained respectively. On the contrary, the reconstruction process is the reverse of decomposition process.

When applying Mallat algorithm to the finite length signal $\left\{c_{N, k}\right\}$, the data beyond the sequence must be extended. Among various boundary extension methods, periodic extension is used frequently. Now, we use the "CDF 9/7" ("bior4.4" in MATLAB) biorthogonal wavelet as an example to illustrate Mallat algorithm, and the length of the 1-D signal $\left\{c_{N, k}\right\}$ is 8 . The coefficients of bior4.4 wavelet filters are

$$
\begin{gathered}
\left\{\tilde{p}_{k}\right\}=\left\{\tilde{p}_{-4} \sim \tilde{p}_{4}\right\}=\left\{\begin{array}{l}
0.0378,-0.0238,-0.1106,0.3774, \\
0.8527,0.3774,-0.1106,-0.0238,0.0378
\end{array}\right\}, \\
\left\{\tilde{q}_{k}\right\}=\left\{\tilde{q}_{-2} \sim \tilde{q}_{4}\right\}=\{-0.0645,0.0407,0.4181,-0.7885,0.4181,0.0407,-0.0645\}, \\
\left\{p_{k}\right\}=\left\{p_{-3} \sim p_{3}\right\}=\{-0.0645,-0.0407,0.4181,0.7885,0.4181,-0.0407,-0.0645\},
\end{gathered}
$$

and

$$
\left\{q_{k}\right\}=\left\{q_{-3} \sim q_{5}\right\}=\left\{\begin{array}{l}
-0.0378,-0.0238,0.1106,0.3774, \\
-0.8527,0.3774,0.1106,-0.0238,-0.0378
\end{array}\right\} .
$$

The processes of biorthogonal wavelet decomposition and reconstruction to 1-D signal $\left\{c_{N, k}\right\}=\{162,163,166,162,155,160,157,161\}$ are shown in Figure 2 and Figure 3 respectively.

\section{The Principle of Windowed APDF Based on DCT}

All phase philosophy can be considered to an application of overlap philosophy [3, 14]. For a digital sequence $\{x(n)\}$, there are $N$ vectors with $N$-dimension. Each vector contains $x(n)$ and has different intercept phases: 


$$
\begin{aligned}
& \boldsymbol{X}_{0}=[x(n), x(n+1), \cdots, x(n+N-2), x(n+N-1)]^{\mathrm{T}}, \\
& \boldsymbol{X}_{1}=z^{-1} \boldsymbol{X}_{0}=[x(n-1), x(n), \cdots, x(n+N-3), x(n+N-2)]^{\mathrm{T}}, \\
& \vdots \\
& \boldsymbol{X}_{N-1}=z^{-(N-1)} \boldsymbol{X}_{0}=[x(n-N+1), x(n-N+2), \cdots, x(n-1), x(n)]^{\mathrm{T}},
\end{aligned}
$$

where $z^{-j}(j=0,1, \cdots, N-1)$ is the delay operator. Obviously, $x(n)$ is the intersection of $\boldsymbol{X}_{i}(i=0,1, \cdots, N-1)$, that is $x(n)=\boldsymbol{X}_{0} \cap \boldsymbol{X}_{1} \cap \cdots \cap \boldsymbol{X}_{N-1}$. According to the regular representation of data matrices, the all phase data matrix of $x(n)$ is defined as $\boldsymbol{X}_{-N+1,0}(n)=\left[\boldsymbol{X}_{0}, \boldsymbol{X}_{1}, \cdots, \boldsymbol{X}_{N-1}\right]$, and all the column vectors $\boldsymbol{X}_{i}(i=0,1, \cdots, N-1)$ span an all phase data space of $x(n)$ [15].

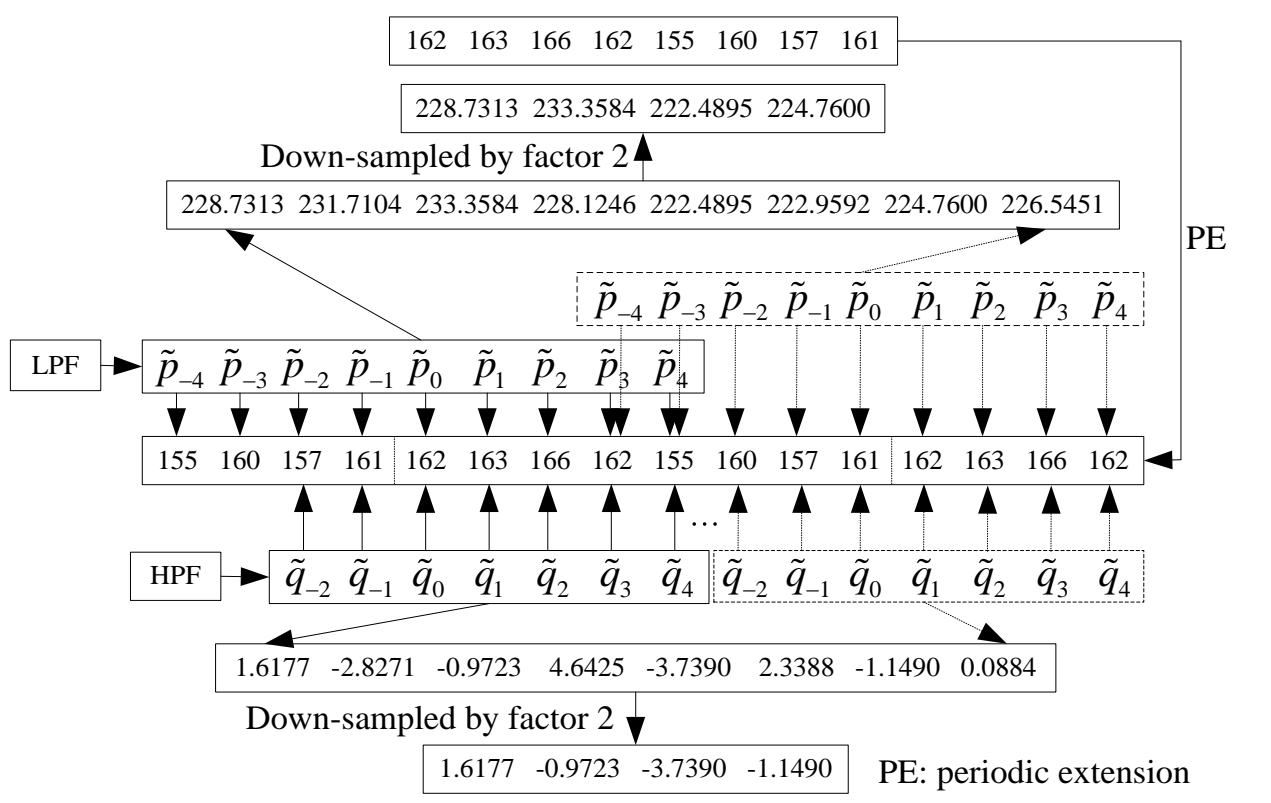

Figure 2. The Decomposition Process of Biorthogonal Wavelet

Architecture of the windowed APDF based on DCT implemented directly in frequency domain is shown in Figure 4 [16]. Windowing is a kind of modulation for the signals. In Figure 4, we add a front window $\boldsymbol{B}^{1}(n) \quad(n=0,1, \cdots, N-1)$ after the input signal $\{x(n)\}$ and a rear window $\boldsymbol{B}^{2}(n)(n=0,1, \cdots, N-1)$ before the output signal $y(n)$ respectively.

$$
\begin{aligned}
\boldsymbol{B}^{1} & =\left[B_{N}^{1}(0), B_{N}^{1}(1), \cdots, B_{N}^{1}(N-1)\right]^{\mathrm{T}}, \\
\boldsymbol{B}^{2} & =\left[B_{N}^{2}(0), B_{N}^{2}(1), \cdots, B_{N}^{2}(N-1)\right]^{\mathrm{T}} .
\end{aligned}
$$

Particularly, when $\boldsymbol{B}^{1}(n)=\mathbf{1}$ and $\boldsymbol{B}^{2}(n)=\mathbf{1}$, the data without window can be regarded as the data with the rectangular window. 


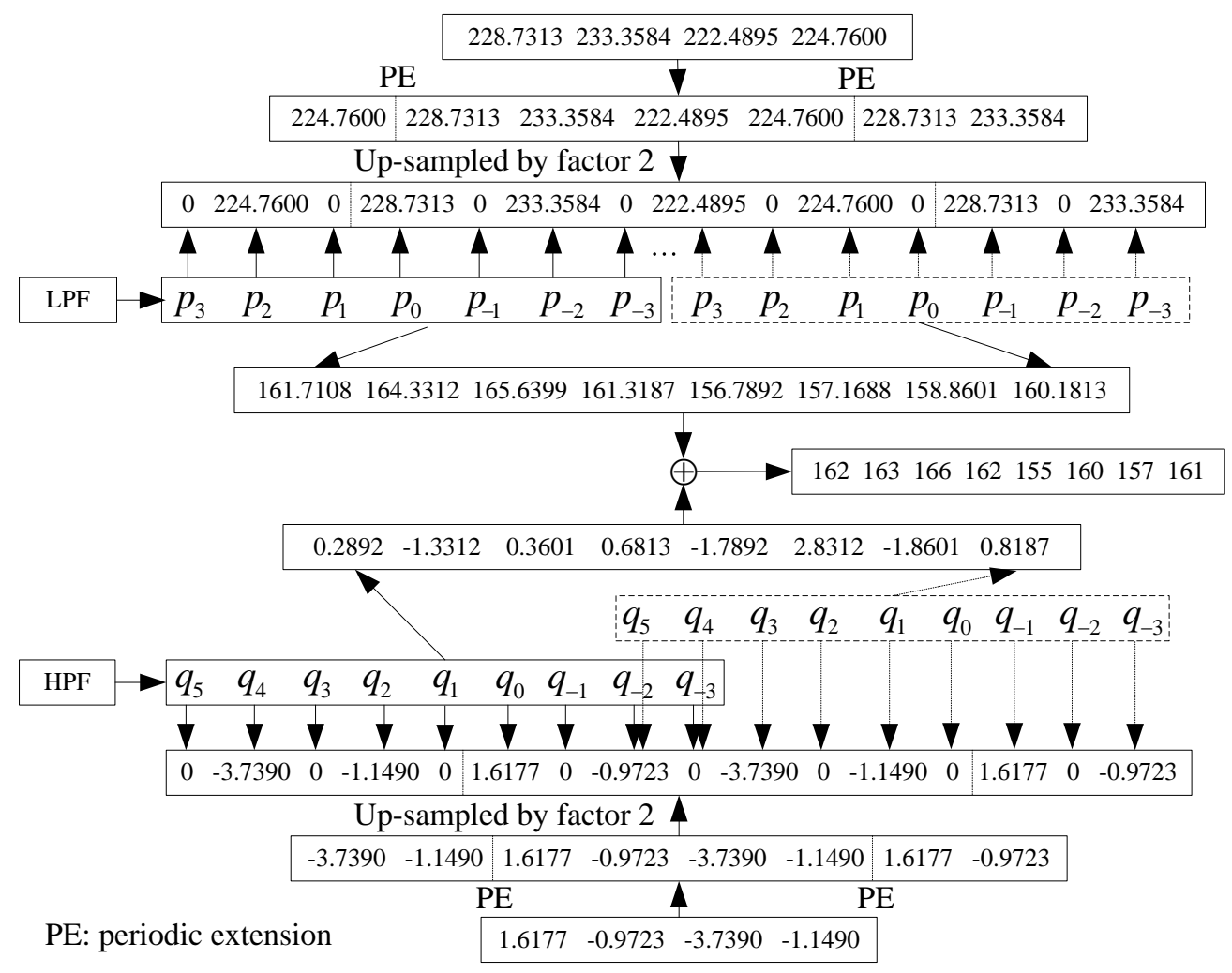

Figure 3. The Reconstruction Process of Biorthogonal Wavelet

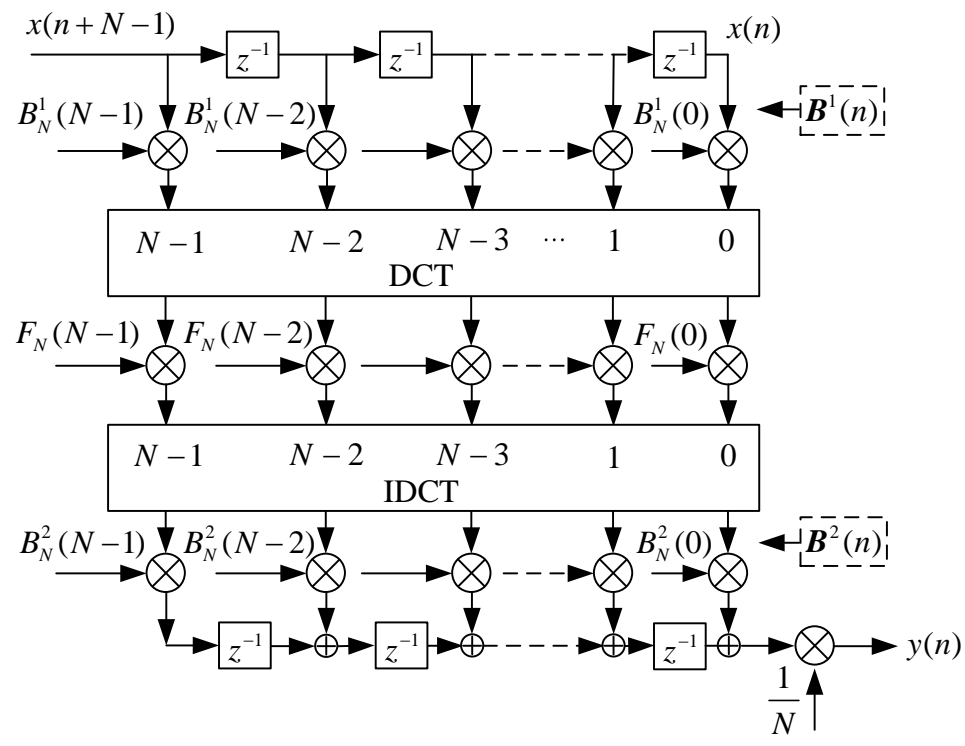

Figure 4. Architecture of the Windowed APDF based on DCT Implemented Directly in Frequency Domain

Denote $\boldsymbol{C}$ as the DCT transform matrix, and $\boldsymbol{C}^{\mathrm{T}}$ is the IDCT transform matrix. $\boldsymbol{C}$ is an $N \times N$ matrix $[C(i, j)]_{N \times N}$, where $C(i, j)=\sqrt{2 / N} K(i) \cos [(2 j+1) i \pi / 2 N]$, 
$K(i)=\left\{\begin{array}{l}1 / \sqrt{2}, i=0 \\ 1, \quad \text { otherwise }\end{array} . \boldsymbol{F}\right.$ is $N$-D respected sequency response vector for windowed APDF based DCT,

$$
\begin{gathered}
\boldsymbol{F}=\left[F_{N}(0), F_{N}(1), \cdots, F_{N}(N-1)\right]^{\mathrm{T}}, \\
\boldsymbol{S}=\left[\boldsymbol{s}_{0}, \boldsymbol{s}_{1}, \cdots, \boldsymbol{s}_{N-1}\right],
\end{gathered}
$$

The matrix $S$ with size of $N \times N$ is the extraction operator, where $s_{i}(i=0,1, \cdots, N-1)$ is the $i$-th $N$-D column vector. The $i$-th element in vector $s_{i}$ is the value 1 and the rest elements are the value 0 .

The relationship between input signal $x(n)$ and output signal $y(n)$ in windowed APDF based on DCT is shown in Figure 5.

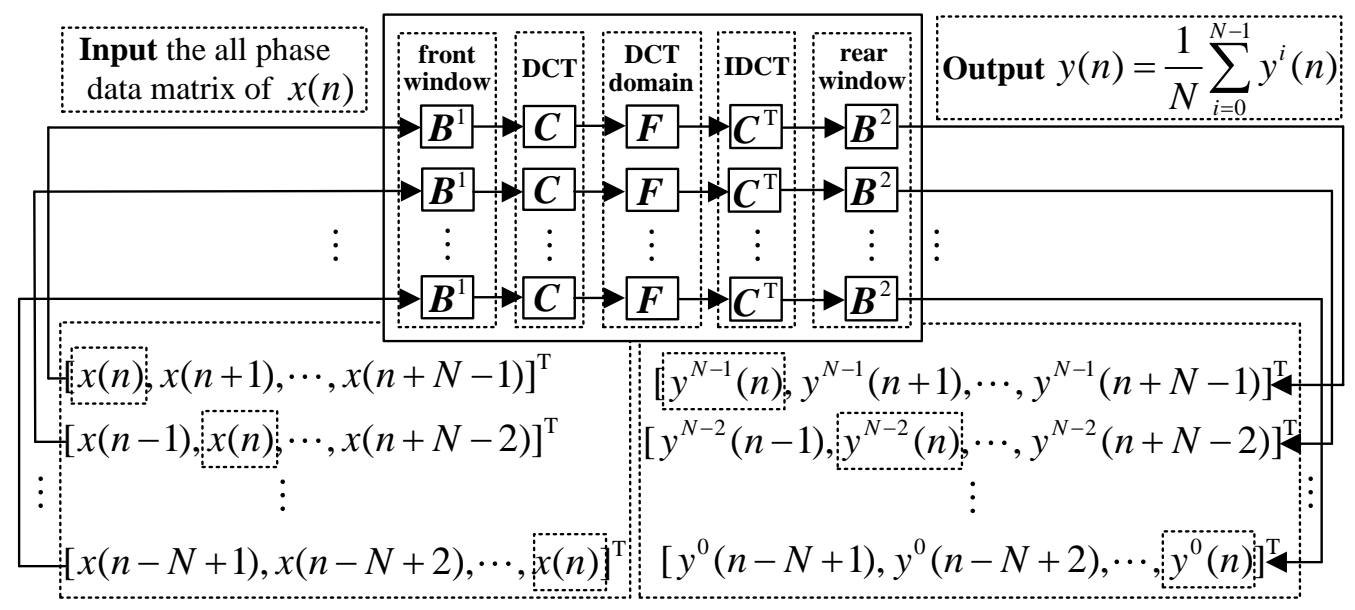

Figure 5. The Relationship between Input Signal $x(n)$ and Output Signal $y(n)$ in Windowed APDF based on DCT

$\boldsymbol{X}_{i}(i=0,1, \cdots, N-1)$ is the $i$-th column vector of the all phase data matrix of $x(n)$. We can get a value $y^{i}(n)$ after $\boldsymbol{X}_{i}$ is filtered. That is

$$
y^{i}(n)=\boldsymbol{s}_{N-1-i}\left\{\boldsymbol{B}^{2} \cdot\left\{\boldsymbol{C}^{\mathrm{T}}\left\{\boldsymbol{F} \cdot\left[\boldsymbol{C}\left(\boldsymbol{B}^{1} \cdot \boldsymbol{X}_{i}\right)\right]\right\}\right\}\right\},
$$

where the mark "." represents dot product operation. According to Eqs. (6) (9) and Eq. (10), we have

$$
\begin{aligned}
y(n) & =\frac{1}{N} \sum_{i=0}^{N-1} y^{i}(n) \\
& =\frac{1}{N} \sum_{i=0}^{N-1}\left\{B_{N}^{2}(N-1-i) \sum_{j=0}^{N-1}\left[\left(\sum_{k=0}^{N-1} F_{N}(k) C_{k, N-1-i} C_{k, j}\right) B_{N}^{1}(j)\right]\right\} x(n+i-N+1+j) \\
& =\sum_{i=0}^{N-1} \sum_{j=0}^{N-1}\left[B_{N}^{2}(i) A(i, j) B_{N}^{1}(j) x(n-i+j)=\sum_{i=0}^{N-1} \sum_{k=-i}^{N-1}\left[B_{N}^{2}(i) A(i, k+i) B_{N}^{1}(k+i) x(n+k)\right]\right. \\
& =h_{0} x(n)+\sum_{k=1}^{N-1}\left[h_{k} x(n+k)+h_{-k} x(n-k)\right],
\end{aligned}
$$


where

$$
\begin{gathered}
A(i, j)=\frac{1}{N} \sum_{m=0}^{N-1} \boldsymbol{F}_{N}(m) \boldsymbol{C}_{m, i} \boldsymbol{C}_{m, j}, \\
h_{k}=\sum_{m=0}^{N-1-k}\left[B_{N}^{2}(m) A(m, m+k) B_{N}^{1}(m+k)\right], h_{-k}=\sum_{m=0}^{N-1-k}\left[B_{N}^{2}(m+k) A(m+k, m) B_{N}^{1}(m)\right] .
\end{gathered}
$$

Eq. (11) can be written in the convolution form:

$$
y(n)=\sum_{k=-(N-1)}^{N-1} h_{k} x(n-k)=h_{n} * x(n) .
$$

From Eq. (14), what we can conclude is that the unit impulse response of $N$-order windowed APDF based on DCT is $\boldsymbol{h}=\left[h_{N-1}, h_{N-2}, \cdots, h_{1}, h_{0}, h_{-1}, \cdots, h_{-N+2}, h_{-N+1}\right]=\left\{h_{k}\right\}$. The $N$-order windowed APDF based on DCT is equivalent to the FIR digital filter with the length of $2 N-1$.

According to Eq. (11), signal filtering in convolution form using APDF based on DCT is shown in Figure 6.

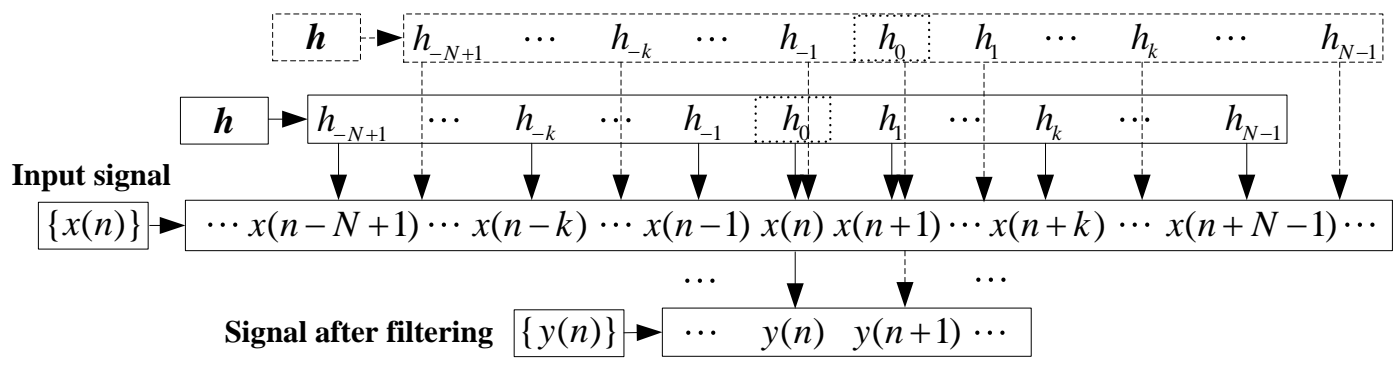

\section{Figure 6. Signal Filtering in Convolution Form using Windowed APDF based on DCT}

Theorem 1: The necessary and sufficient condition of $N$-order windowed APDF based on DCT recognized as the zero-phase digital filters is that the front window and rear window are equal [17]. That is $\boldsymbol{B}^{1}=\boldsymbol{B}^{2} \Leftrightarrow h_{k}=h_{-k}(k=0,1, \cdots, N-1)$.

\section{Algorithm Design of Biorthogonal Wavelet Transform Using Windowed APDF Based on DCT}

\subsection{Relationship between the Coefficients of Biorthogonal Wavelet Filters and Windowed APDF based on DCT}

$\left\{h_{k}\right\} \quad(k=N-1, N-2, \cdots, 1,0,-1, \cdots,-N+2,-N+1)$ are the coefficients of windowed APDF based on DCT. In biorthogonal wavelet transform, the $\left\{\tilde{p}_{k}\right\}$ and $\left\{\tilde{q}_{k}\right\}$ are the coefficients of decomposition filters, while $\left\{p_{k}\right\}$ and $\left\{q_{k}\right\}$ are the coefficients of reconstruction filters. We can find the relationship between $\left\{h_{k}\right\}$ and $\left\{\tilde{p}_{k}\right\},\left\{\tilde{q}_{k}\right\},\left\{p_{k}\right\}$, $\left\{q_{k}\right\}$. These coefficients described above will be discussed as follows. 
Because the length of $N$-order windowed APDF based on DCT is $2 N-1$, the length of the filter coefficients $\left\{\tilde{p}_{k}\right\},\left\{\tilde{q}_{k}\right\},\left\{p_{k}\right\}$, and $\left\{q_{k}\right\}$ should be odd.

Generally speaking, the lengths for biorthogonal wavelet filters $\left\{\tilde{p}_{k}\right\}$ and $\left\{\tilde{q}_{k}\right\}$ are odd or even simultaneously. Reference [13] presented that when the lengths for both of $\left\{\tilde{p}_{k}\right\}$ and $\left\{\tilde{q}_{k}\right\}$ are odd, supposing the support intervals are indicated as

$$
\begin{cases}\operatorname{supp}\left\{\tilde{p}_{k}\right\}=\left[-N_{3}, N_{3}\right], & N_{3}>0, \\ \operatorname{supp}\left\{\tilde{q}_{k}\right\}=\left[-N_{4}+1, N_{4}+1\right], & N_{4}>0,\end{cases}
$$

where

$$
\begin{gathered}
\tilde{p}_{-k}=\tilde{p}_{k}, \quad k=0,1, \cdots, N_{3}, \\
\tilde{q}_{-k}=\tilde{q}_{k+2}, k=-1,0,1, \cdots, N_{4}-1 .
\end{gathered}
$$

Since that

$$
p_{k}=(-1)^{1-k} \tilde{q}_{1-k}, q_{k}=(-1)^{-k} \tilde{p}_{1-k},
$$

correspondingly, the support intervals of $\left\{p_{k}\right\}$ and $\left\{q_{k}\right\}$ can be represented as

$$
\begin{cases}\operatorname{supp}\left\{p_{k}\right\}=\left[-N_{4}, N_{4}\right], & N_{4}>0, \\ \operatorname{supp}\left\{q_{k}\right\}=\left[-N_{3}+1, N_{3}+1\right], & N_{3}>0\end{cases}
$$

where

$$
\begin{gathered}
p_{-k}=p_{k}, \quad k=0,1, \cdots, N_{4}, \\
q_{-k}=q_{k+2}, \quad k=-1,0,1, \cdots, N_{3}-1 .
\end{gathered}
$$

According to Eqs. (17) and (19), windowed APDF based on DCT should be absolutely zero-phase so that the symmetrical coefficients $\left\{h_{k}\right\}$ can correspond to the coefficients $\left\{\tilde{p}_{k}\right\},\left\{\tilde{q}_{k}\right\},\left\{p_{k}\right\}$, and $\left\{q_{k}\right\}$ respectively. That is $h_{k}=h_{-k}$ $(k=0,1, \cdots, N-1)$. With Theorem 1, we get the conclusion that the front window $\boldsymbol{B}^{1}$ and rear window $\boldsymbol{B}^{2}$ of windowed APDF based on DCT should be equal. That is $\boldsymbol{B}^{1}=\boldsymbol{B}^{2}$.

Thus, we can get the following relationship between the coefficients of biorthogonal wavelet filters and windowed APDF based on DCT. As shown in Figure 7, the bior4.4 biorthogonal wavelet $\left(N_{3}=4\right.$ and $N_{4}=3$ ) is taken as an example to illustrate the relationship. $\left\{h_{k}^{i}\right\}(i=1,2,3,4)$ are the coefficients of windowed APDF based on DCT. The low-pass filter $\left\{\tilde{p}_{k}\right\}=\left\{h_{k}^{1}\right\} \quad(k=0, \pm 1, \pm 2, \pm 3, \pm 4)$ and high-pass filter $\left\{\tilde{q}_{k+1}\right\}=\left\{h_{k}^{4}\right\}$ $(k=0, \pm 1, \pm 2, \pm 3)$ are defined as the decomposition filter banks. The low-pass filter $\left\{p_{k}\right\}=\left\{h_{-k}^{2}\right\} \quad(k=0, \pm 1, \pm 2, \pm 3)$ and high-pass filter $\left\{q_{k+1}\right\}=\left\{h_{-k}^{3}\right\} \quad(k=0, \pm 1, \pm 2, \pm 3, \pm 4)$ are defined as the reconstruction filter banks.

Thus, with the convolution filtering method shown in Figure 6, the biorthogonal wavelet transform (shown in Figure 2 and Figure 3 ) to 1-D signal can be realized by using filters $\left\{h_{k}^{i}\right\}$ instead of the coefficients of biorthogonal wavelet filters $\left\{\tilde{p}_{k}\right\},\left\{\tilde{q}_{k}\right\}$, $\left\{p_{k}\right\}$, and $\left\{q_{k}\right\}$. 
The coefficients of filters are symmetrical
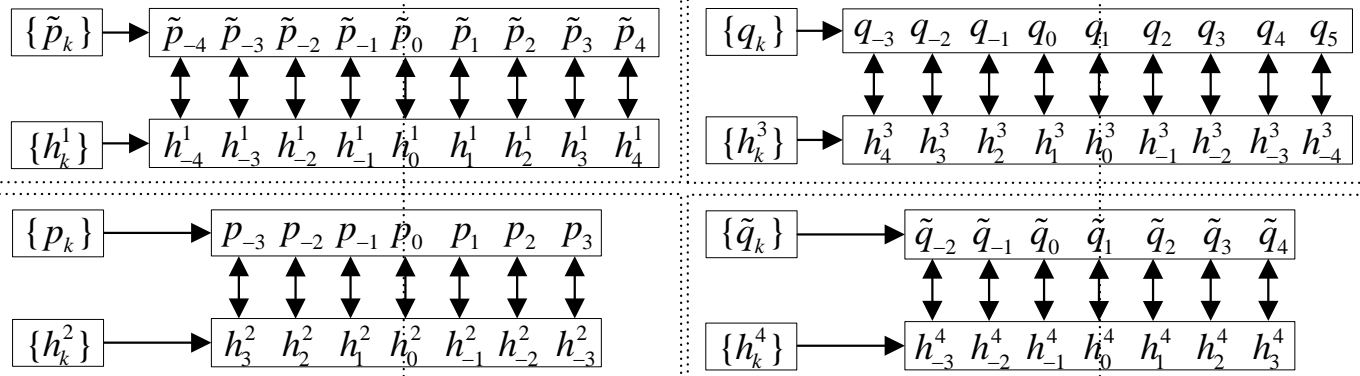

Figure 7. Coefficients $\left\{h_{k}^{i}\right\}$ Correspond to the Coefficients $\left\{\tilde{p}_{k}\right\},\left\{\tilde{q}_{k}\right\},\left\{p_{k}\right\}$, and $\left\{q_{k}\right\}$

\subsection{Process of Input Signal through the Filter}

The algorithm of biorthogonal wavelet transform to 2-D signal using windowed APDF based on DCT is shown in Figure 8.

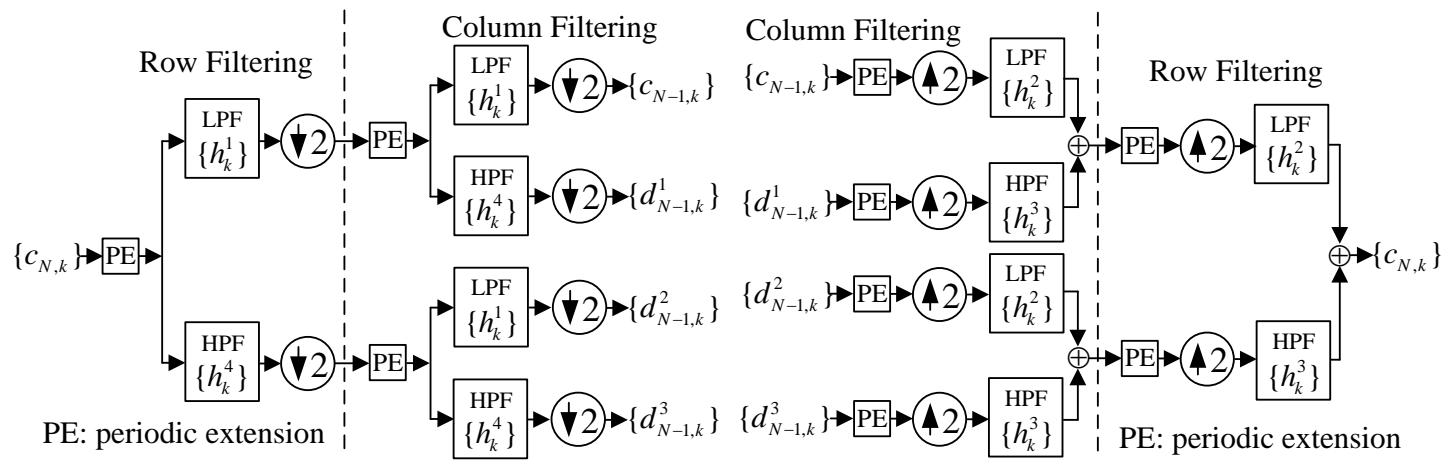

(a)

(b)

\section{Figure 8. Implementation of Biorthogonal Wavelet Transform to 2-D Signal using Windowed APDF based on DCT: (a) Decomposition Algorithm, (b) Reconstruction Algorithm}

The implementation of biorthogonal wavelet decomposition and reconstruction to 2D signal can be decomposed into biorthogonal wavelet decomposition and reconstruction to two 1-D signals. For example, an image signal can be decomposed into two kinds of 1-D signal: row signal and column signal. The process of 1-D signal through the windowed APDF based on DCT is shown in Figure 9.

The process of 1-D signal through the windowed APDF based on DCT can be summarized as the following steps.

Step 1. The input signal $\{x(n)\}$ is extended with periodic extension method.

Step 2. $x(n)$ is a sample of signal $\{x(n)\} . \boldsymbol{X}_{i}$ is the $i$-th column vector in the all phase data matrix of $x(n)$. Each value in $\boldsymbol{X}_{i}$ should be multiplied by each 
corresponding value in the front window $\boldsymbol{B}^{1}$, and denote the result as $\left\{a^{i}\right\} . N$ is the order of the windowed APDF based on DCT.

Step 3. The $\left\{a^{i}\right\}$ is transformed with DCT, and denote the result as $\left\{b^{i}\right\}$.

Step 4. $\boldsymbol{F}$ is the sequency response vector in the windowed APDF based on DCT. Each value in $\left\{b^{i}\right\}$ should be multiplied by each corresponding value in the response vector $\boldsymbol{F}$, and the result is denoted as $\left\{c^{i}\right\}$.

Step 5. The $\left\{c^{i}\right\}$ is transformed with IDCT, and the result is denoted as $\left\{d^{i}\right\}$.

Step 6. Each value in $\left\{d^{i}\right\}$ should be multiplied by each corresponding value in the rear window $\boldsymbol{B}^{2}$, and the result is denoted as $\left\{e^{i}\right\}$.

Step 7. Extract the $(N-i)$-th element in the vector $\left\{e^{i}\right\}$, and the extracted element is the output value $y^{i}(n)$.

Step 8. Because there are $N$ column vectors in the all phase data matrix of $x(n)$, $y^{i}(n), i=0,1, \cdots, N-1$ can be obtained. We take the mean of the values $y^{i}(n)$ as the filtering output that corresponding to the input value $x(n)$, and the output is denoted as $y(n)$.

Step 9. Repeating Step $2 \sim$ Step 8 until the entire output signal $\{y(n)\}$ is obtained.

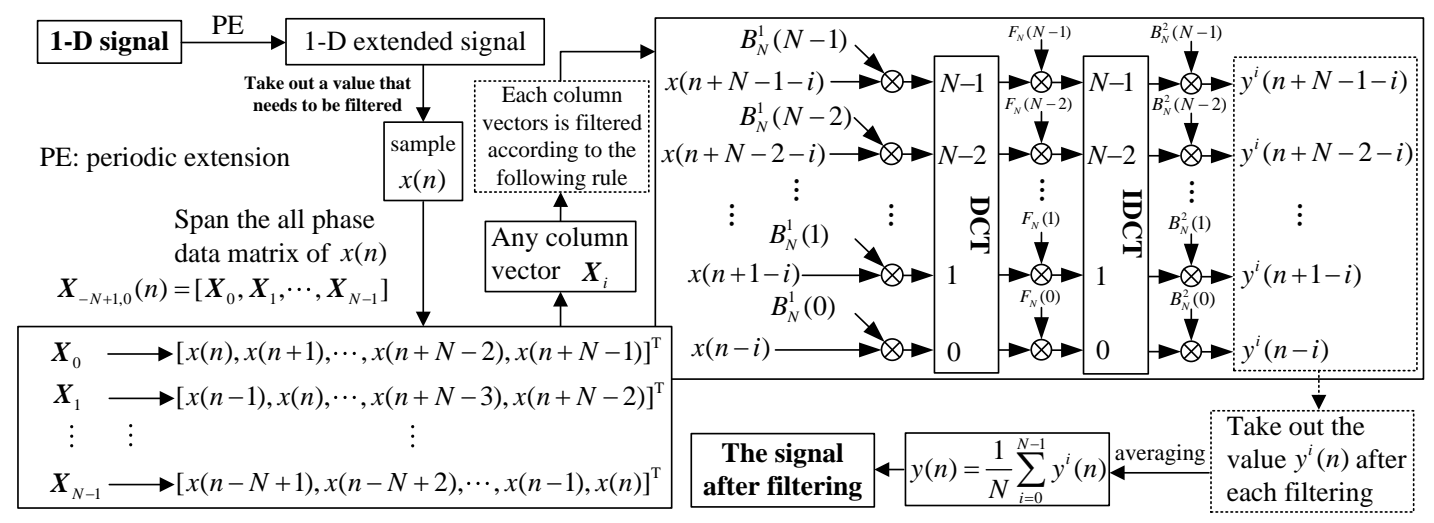

Figure 9. The process of 1-D signal through the windowed APDF based on DCT

\subsection{Solving Sequency Response Vector and Window Sequences in the Windowed APDF based on DCT}

The coefficients $\left\{\tilde{p}_{k}\right\},\left\{\tilde{q}_{k}\right\},\left\{p_{k}\right\}$, and $\left\{q_{k}\right\}$ in biorthogonal wavelet transform have been obtained. The sequency response vector $\boldsymbol{F}$ and window sequences $\left(\boldsymbol{B}^{1}, \boldsymbol{B}^{2}\right)$ in the windowed APDF based on DCT can be designed according to the relationship that the coefficients of the windowed APDF based on DCT should correspond to the coefficients of biorthogonal wavelet filters. 
As discussed above, the front window $\boldsymbol{B}^{1}$ and the rear window $\boldsymbol{B}^{2}$ are the same. That is $\boldsymbol{B}^{1}=\boldsymbol{B}^{2}=\boldsymbol{B}=\left[B_{N}(0), B_{N}(1), \cdots, B_{N}(N-1)\right]^{\mathrm{T}}$.

Denote the $f_{N}(k)$ as IDCT-like spectrum of the sequency response vector $\boldsymbol{F}$, that is

$$
\boldsymbol{f}_{N}=\boldsymbol{r}_{N} \boldsymbol{F},
$$

where $\boldsymbol{r}_{N}$ is defined as an $(N+1) \times N$ IDCT-like matrix:

$$
r_{N}(i, j)=\left\{\begin{array}{lc}
\sqrt{\frac{1}{2 N}}, & j=0,0 \leq i \leq N, \\
\sqrt{\frac{2}{N}} \cos \frac{i j \pi}{N}, & 1 \leq j \leq N-1,0 \leq i \leq N .
\end{array}\right.
$$

So far, we can obtain the following conclusions [7]. Do not care about whether $N$ is odd or even. If $k(k=0,-1, \cdots,-N+1)$ and $N$ have the same parity, we have

$$
\begin{aligned}
h_{k}= & \frac{1}{N \sqrt{2 N}} \sum_{l=0}^{(N+k-2) / 2} f_{N}(2 l-k+1)\left[B_{N}(l-k) B_{N}(l)+B_{N}(N-1-l) B_{N}(N-1-l+k)\right] \\
& +\frac{1}{N \sqrt{2 N}}\left[\sum_{l=-k}^{N-1} B_{N}(l) B_{N}(l+k)\right] f_{N}(-k) .
\end{aligned}
$$

If $k$ and $N$ have different parity, we have

$$
\begin{aligned}
h_{k}= & \frac{1}{N \sqrt{2 N}} \sum_{l=0}^{(N+k-3) / 2} f_{N}(2 l-k+1)\left[B_{N}(l-k) B_{N}(l)+B_{N}(N-1-l) B_{N}(N-1-l+k)\right] \\
& +f_{N}(N) B_{N}\left(\frac{N+k-1}{2}+k\right) B_{N}\left(\frac{N+k-1}{2}\right)+\frac{1}{N \sqrt{2 N}}\left[\sum_{l=-k}^{N-1} B_{N}(l) B_{N}(l+k)\right] f_{N}(-k) .
\end{aligned}
$$

Obviously, $h_{k}(k=N-1, N-2, \cdots, 0)$ can be obtained according to Eq. (24):

$$
h_{k}=h_{-k} \text {. }
$$

If we know the coefficients $h_{k}(k=N-1, N-2, \cdots, 1,0,-1, \cdots,-N+2,-N+1)$ of the windowed APDF based on DCT, according to Eqs. (20) (24), eligible sequency response vector $\boldsymbol{F}$ and eligible window sequence $\boldsymbol{B}$ can be obtained by solving nonlinear equations.

\section{Experimental Results}

The principle and implementation of the biorthogonal wavelet transform using windowed APDF based on DCT have been described above. As a verification of the algorithm, in the following part, typical test images are used as input signal to simulate the process of wavelet transform in computer by MATLAB 7.0. This paper selects the biorthogonal wavelet "CDF 9/7" as an example to explain the process of transformation. This process is also called the separable multi-resolution analysis of 2-D images, which has been shown in Figure 8.

Windowed APDF based on DCT is used to realize 2-D signal biorthogonal wavelet decomposition and reconstruction in Figure 8. The next work we should do is to solve 
the sequency response vector $\boldsymbol{F}$ and window sequence $\boldsymbol{B}$ in each windowed APDF based on DCT.

As shown in Figure 7, low-pass filter $\left\{h_{k}^{1}\right\}$ corresponds to $\left\{\tilde{p}_{k}\right\}$,

$$
\begin{aligned}
\left\{h_{k}^{1}\right\}=\left\{\tilde{p}_{k}\right\}=\{ & 0.85269867900889,0.37740285561283,-0.11062440441844, \\
& -0.02384946501956,0.03782845550726\}^{\mathrm{T}}, k=0,-1,-2,-3,-4 .
\end{aligned}
$$

Then $\left\{h_{k}^{1}\right\}(k=4,3,2,1)$ can be obtained by symmetry.

According to Eqs. (20) (24), eligible sequency response vector $\boldsymbol{F}$ and eligible window sequence $\boldsymbol{B}$ can be obtained by solving nonlinear equations. That is

$$
\begin{aligned}
\boldsymbol{F}= & {[1.11475331542401,0.988891664170742,} \\
& 1.20722820574485,0.207996712645094,-0.234650381462672]^{\mathrm{T}}, \\
\boldsymbol{B}= & {[1.14800813102789,1.06165266504137,} \\
& 1.21391031718411,1.06165266504137,1.14800813102789]^{\mathrm{T}} .
\end{aligned}
$$

The other three cases follow the same principle, and all the results are shown in Table $1 \sim$ Table 4.

\section{Table 1. Low-pass Decomposition Filter Parameters of the Windowed APDF based on DCT}

\begin{tabular}{|c|c|c|c|}
\hline \multirow{2}{*}{$\begin{array}{c}\text { Reconstruction } \\
\text { filter index } k\end{array}$} & \multicolumn{3}{|c|}{ Low-pass filter $\left\{h_{k}^{1}\right\}$ corresponds to $\left\{\tilde{p}_{k}\right\}$} \\
\cline { 2 - 4 } & $\begin{array}{c}\left\{h_{k}^{1}\right\}\left(k>0,\left\{h_{k}^{1}\right\} \text { is }\right. \\
\text { obtained by symmetry })\end{array}$ & $\begin{array}{c}\text { Sequency response } \\
\text { vector } \boldsymbol{F}\end{array}$ & $\begin{array}{c}\text { Window sequences } \\
\boldsymbol{B}\end{array}$ \\
\hline 0 & 0.85269867900889 & 1.11475331542401 & 1.14800813102789 \\
\hline-1 & 0.37740285561283 & 0.988891664170742 & 1.06165266504137 \\
\hline-2 & -0.11062440441844 & 1.20722820574485 & 1.21391031718411 \\
\hline-3 & -0.02384946501956 & 0.207996712645094 & 1.06165266504137 \\
\hline-4 & 0.03782845550726 & -0.234650381462672 & 1.14800813102789 \\
\hline
\end{tabular}

\section{Table 2. High-pass Decomposition Filter Parameters of the Windowed APDF based on DCT}

\begin{tabular}{|c|c|c|c|}
\hline \multirow{2}{*}{$\begin{array}{c}\text { Reconstruction } \\
\text { filter index } k\end{array}$} & \multicolumn{3}{|c|}{ High-pass filter $\left\{h_{k}^{4}\right\}$ corresponds to $\left\{\tilde{q}_{k}\right\}$} \\
\cline { 2 - 4 } & $\begin{array}{c}\left\{h_{k}^{4}\right\}\left(k>0,\left\{h_{k}^{4}\right\} \text { is }\right. \\
\text { obtained by symmetry })\end{array}$ & $\begin{array}{c}\text { Sequency response } \\
\text { vector } \boldsymbol{F}\end{array}$ & $\begin{array}{c}\text { Window sequences } \\
\boldsymbol{B}\end{array}$ \\
\hline 0 & -0.78848561640558 & 0.178348726516796 & -1.17856456042842 \\
\hline-1 & 0.41809227322162 & -0.161609767534304 & -0.630328266513568 \\
\hline-2 & 0.04068941760916 & -1.94160159411144 & -0.630328266497937 \\
\hline-3 & -0.06453888262870 & -2.53999394876350 & -1.17856456037055 \\
\hline
\end{tabular}


Table 3. Low-pass Reconstruction Filter Parameters of the Windowed APDF based on DCT

\begin{tabular}{|c|c|c|c|}
\hline \multirow{2}{*}{$\begin{array}{c}\text { Reconstruction } \\
\text { filter index } k\end{array}$} & \multicolumn{3}{|c|}{ Low-pass filter $\left\{h_{k}^{2}\right\}$ corresponds to $\left\{p_{k}\right\}$} \\
\cline { 2 - 4 } & $\begin{array}{c}\left\{h_{k}^{2}\right\}\left(k>0,\left\{h_{k}^{2}\right\} \text { is }\right. \\
\text { obtained by symmetry })\end{array}$ & $\begin{array}{c}\text { Sequency response } \\
\text { vector } \boldsymbol{F}\end{array}$ & $\begin{array}{c}\text { Window sequences } \\
\boldsymbol{B}\end{array}$ \\
\hline 0 & 0.78848561640558 & 1.06031621715721 & 1.16082955466259 \\
\hline-1 & 0.41809227322162 & 1.25876211576288 & 1.14893864243466 \\
\hline-2 & -0.04068941760916 & 0.257099561729294 & 1.14893864183989 \\
\hline-3 & -0.06453888262870 & -0.222318332512497 & 1.16082955431201 \\
\hline
\end{tabular}

Table 4. High-pass Reconstruction Filter Parameters of the Windowed APDF based on DCT

\begin{tabular}{|c|c|c|c|}
\hline \multirow{2}{*}{$\begin{array}{c}\text { Reconstruction } \\
\text { filter index } k\end{array}$} & \multicolumn{3}{|c|}{ High-pass filter $\left\{h_{k}^{3}\right\}$ corresponds to $\left\{q_{k}\right\}$} \\
\cline { 2 - 4 } & $\begin{array}{c}\left\{h_{k}^{3}\right\}\left(k>0,\left\{h_{k}^{3}\right\} \text { is }\right. \\
\text { obtained by symmetry })\end{array}$ & $\begin{array}{c}\text { Sequency response } \\
\text { vector } \boldsymbol{F}\end{array}$ & $\begin{array}{c}\text { Window sequences } \\
\boldsymbol{B}\end{array}$ \\
\hline 0 & -0.85269867900889 & 0.231796375205308 & -1.42668294066615 \\
\hline-1 & 0.37740285561283 & -0.101299026664208 & -0.930583623766868 \\
\hline-2 & 0.11062440441844 & -1.37397869330961 & -0.305928073054824 \\
\hline-3 & -0.02384946501956 & -1.66297911700527 & -0.930583627855862 \\
\hline-4 & -0.03782845550726 & -1.20535892515458 & -1.42668294066667 \\
\hline
\end{tabular}

The multi-level decomposition and reconstruction are not the focus of this paper, so we take only one layer wavelet transform to illustrate the feasibility of the proposed algorithm. The test image is the MATLAB image "woman2.mat" whose size is $128 \times 128$. The result of one layer wavelet transform to test image "woman2" applying windowed APDF based on DCT is shown in Figure 10, and the result of one layer wavelet transform to test image "woman2" applying biorthogonal wavelet is shown in Figure 11.

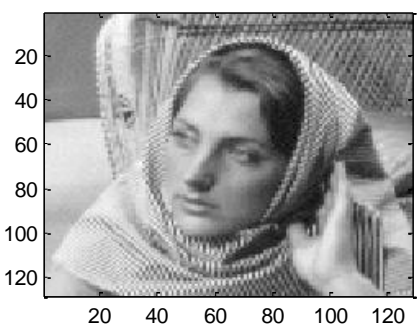

(a)

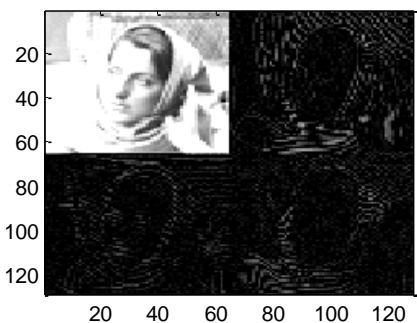

(b)

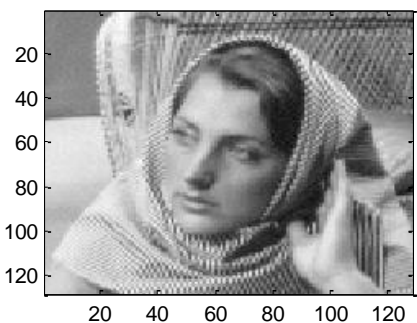

(c)

Figure 10. Implementation of Decomposition and Reconstruction using Windowed APDF based on DCT: (a) Original Image, (b) Decomposition, (c) Reconstruction. 


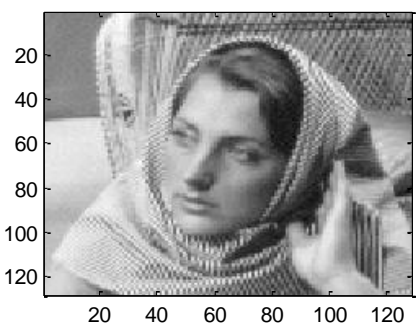

(a)

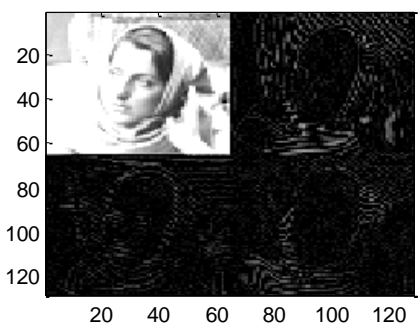

(b)

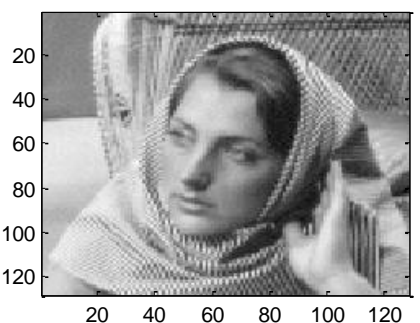

(c)

Figure 11. The Decomposition and Reconstruction Results using the Biorthogonal Wavelet: (a) Original Image, (b) Decomposition, (c) Reconstruction

The methods of windowed APDF based on DCT and the biorthogonal wavelet have similar results. The mean squared error (MSE) of the reconstruction and decomposition is $1.9943 \times 10^{-21}$ applying windowed APDF based on DCT, and the MSE of the reconstruction and decomposition is $1.9896 \times 10^{-21}$ applying biorthogonal wavelet.

\section{Conclusion}

On the basis of the biorthogonal wavelet transform and the windowed APDF based on DCT, this paper proposes a new algorithm to implement biorthogonal wavelet transform using the all phase digital filter. The experiments to typical test images are done in MATLAB. Experimental results show that whether using the windowed APDF based on DCT or the biorthogonal wavelet, the results of decomposition and reconstruction perform very well and are almost the same. So we can draw a conclusion that for biorthogonal wavelets whose filter coefficients length are odd, their wavelet transforms can be implemented using the windowed APDF based on DCT.

With the windowed APDF based on DCT designed in this paper, more applications in digital image processing, such as image coding, image denoising, and image edge detection can be realized easily. These issues could be resolved in future research.

\section{Acknowledgements}

This work was supported by the Fundamental Research Funds of Shandong University (Grant No. 2014ZQXM008), the Graduate Innovation Foundation of Shandong University, WeiHai (GIFSDUWH) (Grant No. yjs13022, yjs13025), and the promotive research fund for excellent young and middle-aged scientists of Shandong Province, China (Grant No. BS2013DX022). The authors would like to thank Xiaoyan Wang for her kind help and valuable suggestions. The authors also thank the anonymous reviewers and the editors for their valuable comments to improve the presentation of the paper.

\section{References}

[1] A. V. Oppenheim and R. W. Schafer, "Discrete-Time Signal Processing", Prentice-Hall, Inc., New Jersey, (1989), pp. 313-317, 444-488.

[2] Z. X. Hou and X. Yang, "The all phase DFT filter", in: Proc. of the 10th IEEE Digital Signal Processing Workshop and the 2nd IEEE Signal Processing Education Workshop, Pine Mountain, Georgia, USA, (2002), October 13-16, pp. 221-226.

[3] Z. X. Hou, Z. H. Wang and X. Yang, "Design and implementation of all phase DFT digital filter", Acta Electronica Sinica, vol. 31, no. 4, (2003), pp. 539-543. (in Chinese) 
[4] Z. X. Hou, "Design and application of the discrete cosine sequency filters", Journal of Tianjin University (Science and Technology), vol. 32, no. 3, (1999), pp. 324-328. (in Chinese)

[5] Z. X. Hou, X. J. Guo, and Xi Yang, "A hierarchical coding technique based on all phase IDCT interpolation", Journal of Electronics and Information Technology, vol. 27, no. 6, (2005), pp. 865-869. (in Chinese)

[6] B. Shen, I. K. Sethi, and V. Bhaskaran, "DCT convolution and its application in compressed domain", IEEE Trans. on Circuits and Systems for Video Technology, vol. 8, no. 8, (1998), pp. 947-952.

[7] L. L. Zhao, “All phase digital filtering and image interpolation based on IDCT/DCT", Ph.D. Thesis, Tianjin University, China, (2006), pp. 49-62. (in Chinese)

[8] S. G. Mallat, "Multiresolution approximations and wavelet orthonormal bases of $\boldsymbol{L}^{2}(\boldsymbol{R})$ ", Trans. of the American Mathematical Society, vol. 315, no. 1, (1989), pp. 69-87.

[9] D. L. Donoho, "De-noising by soft-thresholding", IEEE Trans. on Information Theory, vol. 41, no. 3, (1995), pp. 613-627.

[10] D. Taubman, "High performance scalable image compression with EBCOT", IEEE Trans. on Image Processing, vol. 9, no. 7, (2000), pp. 1158-1170.

[11] S. G. Mallat, "A theory for multiresolution signal decomposition: the wavelet representation", IEEE Trans. on Pattern Analysis and Machine Intelligence, vol. 11, no. 7, (1989), pp. 674-693.

[12] B. C. Jiang, A. P. Yang, C. Y. Wang, and Z. X. Hou, "Implementation of biorthogonal wavelet transform using discrete cosine sequency filter", International Journal of Signal Processing, Image Processing and Pattern Recognition, vol. 6, no. 4, (2013), pp. 179-189.

[13] C. Y. Wang, "Research on all phase biothogonal transform theory and its applications in image coding", Ph.D. Thesis, Tianjin University, China, (2010), pp. 25-28, 106-114. (in Chinese)

[14] Z. H. Wang and X. D. Huang, "All Phase Spectrum Analysis and Digital Signal Filtering Technology", Publishing House of Electronics Industry, Beijing, China, (2009), pp. 1-8. (in Chinese)

[15] Z. X. Hou, "Design and implementation of the all phase sequency filter", Signal Processing, vol. 17, supplement, (2001), pp. 132-135. (in Chinese)

[16] Z. X. Hou and N. N. Xu, "Windowed all phase DFT digital filter", Journal of Tianjin University (Science and Technology), vol. 38, no. 5, (2005), pp. 448-454. (in Chinese)

[17] F. Su and Z. H. Wang, "Implementation and design of all phase FIR filter in DCT domain", Journal of Tianjin University (Science and Technology), vol. 37, no. 12, (2004), pp. 1110-1114. (in Chinese)

\section{Authors}

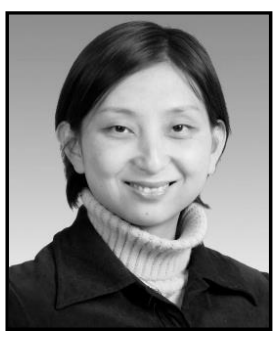

Xiao Zhou, she was born in Shandong province, China in 1982. She received her B.E. degree in automation from Nanjing University of Posts and Telecommunications, China, in 2003, her M.E. degree in information and communication engineering from Inha University, Korea in 2005, and her Ph.D. degree in information and communication engineering from Tsinghua University, China in 2013. Now she is a lecturer in the School of Mechanical, Electrical and Information Engineering, Shandong University, Weihai, China. Her current research interests include wireless communication technology, image processing and transmission technology.

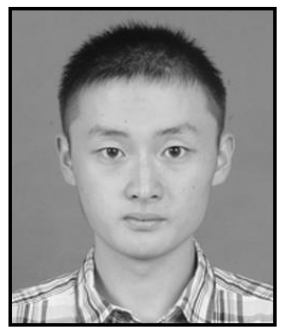

Qiming Fu, he was born in Jiangxi province, China in 1992. He received his B.S. degree in electronic information science and technology from Shandong University, Weihai, China, in 2013. Now he is pursuing his M.E. degree in electronics and communication engineering in Shandong University, Weihai, China. His research interests concentrate on image and video processing. 


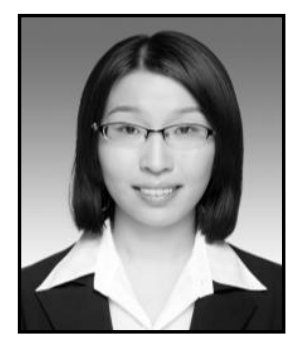

Fanfan Yang, she was born in Shandong province, China in 1989. She received her B.E. degree in communication engineering from Shandong University, Weihai, China, in 2013. Now she is pursuing her M.E. degree in communication and information system in Shandong University, Weihai, China. Her research interests concentrate on image and video processing.

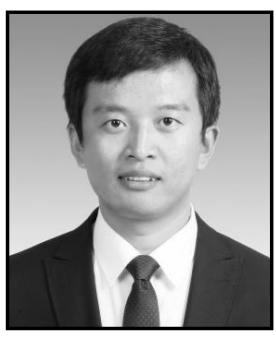

Chengyou Wang, he was born in Shandong province, China in 1979. He received his B.E. degree in electronic information science and technology from Yantai University, China, in 2004 and his M.E. and $\mathrm{Ph} . \mathrm{D}$. degree in signal and information processing from Tianjin University, China, in 2007 and 2010 respectively. Now he is an associate professor in the School of Mechanical, Electrical and Information Engineering, Shandong University, Weihai, China. His current research interests include image processing and transmission technology, multidimensional signal and information processing, and smart grid technology. 